or TCF-1, a transcription factor related to progenitor-like exhausted T cells. In ex vivo functional assays, anti-PD-1 treatment increased the production of IFN- $\square$ and TNF, and the expression of a proliferation marker, Ki-67 among tumor-infiltrating $\mathrm{CD} 8+\mathrm{T}$ cells. Interestingly, the effects of anti-PD-1 treatment were further enhanced by the combination treatment with CMPD0914.

Conclusions In summary, we demonstrated that HPK1 and SLP76 are expressed by human tumor-infiltrating T cells, particularly PD-1brightCD $8+\mathrm{T}$ cells, and that anti-PD-1-induced T-cell reinvigoration is significantly enhanced by an HPK1 inhibitor, CMPD0914, rationalizing the combination of antiPD1/PD-L1 and HPK1 inhibitors for the treatment of cancer.

http://dx.doi.org/10.1136/jitc-2020-SITC2020.0859

\section{TARGETING IMMUNOSUPPRESSIVE MACROPHAGES OVERCOMES PARP-INHIBITOR RESISTANCE IN BRCA1- ASSOCIATED TRIPLE-NEGATIVE BREAST CANCER}

\begin{abstract}
${ }^{1}$ Anita Mehta*, ${ }^{1}$ Emily Cheney, ${ }^{1}$ Christina Hartl, ${ }^{1}$ Constantia Pantelidou, ${ }^{2}$ Madison Oliwa, ${ }^{1}$ Jessica Castrillon, ${ }^{3}$ Jia-Ren Lin, ${ }^{4}$ Katie Hurst, ${ }^{5}$ Mateus de Oliveira Taveira, ${ }^{3}$ Nathan Johnson, ${ }^{6}$ William Oldham, ${ }^{3}$ Marian Kalocsay, ${ }^{3}$ Matthew Berberich, ${ }^{3}$ Sarah Boswell, ${ }^{1}$ Aditi Kothari, ${ }^{1}$ Shawn Johnson, ${ }^{7}$ Deborah Dillon, ${ }^{7}$ Mikel Lipschitz, ${ }^{7}$ Scott Rodig, ${ }^{7}$ Sandro Santagata, ${ }^{1}$ Judy Garber, ${ }^{8}$ Nadine Tung, ${ }^{9}$ José Yélamos, ${ }^{4}$ Jessica Thaxton, ${ }^{1}$ Elizabeth Mittendorf, ${ }^{3}$ Peter Sorger, ${ }^{1}$ Geoffrey Shapiro, 'Jennifer Guerriero. 'Dana Farber Cancer Institute, Boston, MA, USA; ${ }^{2}$ Dana-Farber Cancer Institute and Harvard, Boston, USA; ${ }^{3}$ Harvard Medical School, Boston, MA, USA; ${ }^{4}$ Medical University of South Carolina, Charleston, SC, USA; ${ }^{5}$ A. C. Camargo Cancer Center, São Paulo, Brazil; ' ${ }^{B}$ 'righam and Women's Hospital and Harvard, Boston, MA, USA; ' Brigham and Women's Hospital and Harvard, Boston, MA, USA; ${ }^{8}$ Beth Israel Deaconess Medical Center, Boston, MA, USA; ${ }^{9}$ Medical Research Institute, Barcelona, Spain
\end{abstract}

Background Despite objective responses to PARP inhibition and improvements in progression-free survival compared to standard chemotherapy in patients with BRCA-associated triple-negative breast cancer (TNBC), benefits are transitory.

Methods Using high dimensional single-cell profiling of human TNBC, here we demonstrate that macrophages are the predominant infiltrating immune cell type in BRCA-associated TNBC. Through multi-omics profiling we show that PARP inhibitors enhance both anti- and pro-tumor features of macrophages through glucose and lipid metabolic reprogramming driven by the sterol regulatory element-binding protein 1 (SREBP-1) pathway.

Results Combined PARP inhibitor therapy with CSF-1R blocking antibodies significantly enhanced innate and adaptive antitumor immunity and extends survival in BRCA-deficient tumors in vivo and is mediated by CD8 + T-cells.

Conclusions Collectively, our results uncover macrophage-mediated immune suppression as a liability of PARP inhibitor treatment and demonstrate combined PARP inhibition and macrophage targeting therapy induces a durable reprogramming of the tumor microenvironment, thus constituting a promising therapeutic strategy for TNBC.

http://dx.doi.org/10.1136/jitc-2020-SITC2020.0860

\section{DEVELOPMENT OF FPA157, AN ANTI-CCR8 DEPLETING ANTIBODY ENGINEERED TO PREFERENTIALLY ELIMINATE TUMOR-INFILTRATING T REGULATORY CELLS}

Andrew Rankin, Edwina Naik*. Five Prime Therapeutics, South San Francisco, CA, USA
Background The clinical success of PD-1- and CTLA-4immune checkpoint inhibitors highlights the key contribution of immunosuppression to limiting effective anti-tumor responses. However, as many patients do not respond to antiPD1 or CTLA4 therapy ${ }^{1-3}$ novel therapeutics that target additional immune-suppressive mechanisms are needed. Regulatory $\mathrm{T}$ cells (Tregs) inhibit immune responses in the tumor microenvironment via multiple suppressive mechanisms. ${ }^{4} 5$ Existing Treg-targeting agents lack specificity for intratumoral Tregs and can also deplete effector cells, a property that has likely contributed to the lack of clinical activity observed to date. CCR8 (C-C chemokine receptor 8) is selectively expressed on highly activated intratumoral Tregs, its high expression correlates with poor prognosis in multiple human tumor types ${ }^{6} 7$ and depletion of CCR8 + Tregs in preclinical models elicited potent anti-tumor activity. These observations provided rationale for the development of a CCR8-specific human depleting antibody.

Methods Human FOXP3 and CCR8 expression was correlated across multiple tumor types using TCGA datasets and expression of CCR8 evaluated in primary tumor explants and PBMCs by flow cytometry. The efficacy of anti-CCR 8 antibody treatment was evaluated in the MC38 and CT26 murine tumor models. The depletion of Tregs following anti-CCR8 treatment was assessed by flow cytometry. Flow cytometricbased binding assays were performed using cell lines expressing human or cynomolgus CCR8. Purified human NK cells were co-cultured with CCR8 + target cells and flow cytometry used to evaluate antibody-dependent killing activity.

Results CCR8 expression was highly correlated with FoxP3 across multiple cancer subtypes and was low to absent on effector $\mathrm{T}$ cells. Importantly, CCR8 was not detected on any peripheral human leukocyte subset. In murine tumor models, anti-CCR8 antibody treatment reduced tumor growth in a dose- and Fc-gamma-receptor-dependent manner and resulted in complete regressions and the development of memory. Tumor shrinkage was associated with a reduction in intratumoral Tregs and increased representation of intratumoral CD8 T cells. FPA157 is a highly specific human and cynomolgus crossreactive CCR8 antibody that does not bind closely related chemokine receptors. FPA157 was engineered to enhance antibody-dependent cell-mediated cytotoxicity (eADCC) and elicited potent NK-mediated killing of target cells expressing CCR8 at levels observed on human intratumoral

Tregs.

Conclusions FPA157 is a CCR8-specific monoclonal antibody with eADCC activity that is being developed for the treatment of cancer. Depletion of CCR8 + Tregs induced substantial antitumor activity in pre-clinical models, thus supporting the clinical evaluation of FPA157 as a novel approach to alleviate immune suppression in the microenvironment of human solid tumors.

\section{REFERENCES}

1. Hellmann MD, Ciuleanu TE, Pluzanski A, Lee JS, Otterson GA, Audigier-Valette C, Minenza $E$, Linardou $H$, Burgers $S$, Salman $P$, Borghaei $H$, Ramalingam SS, Brahmer J, Reck M, O'Byrne KJ, Geese WJ, Green G, Chang H, Szustakowski J, Bhagavatheeswaran P, Healey D, Fu Y, Nathan F, Paz-Ares L. Nivolumab plus Ipilimumab in lung cancer with a high tumor mutational burden. $N$ Engl J Med 2018:378(22):2093-2104.

2. Wolchok JD, Chiarion-Sileni V, Gonzalez R, Rutkowski P, Grob JJ, Cowey CL, Lao CD, Wagstaff J, Schadendorf D, Ferrucci PF, Smylie M, Dummer R, Hill A, Hogg D, Haanen J, Carlino MS, Bechter O, Maio M, Marquez-Rodas I, Guidoboni M, McArthur G, Lebbé C, Ascierto PA, Long GV, Cebon J, Sosman J, Postow MA, Callahan MK, Walker D, Rollin L, Bhore R, Hodi FS, Larkin J. Overall survival with 